\title{
Visit-to-visit variability of glycemia and vascular complications: the Hoorn Diabetes Care System cohort
}

\author{
Roderick C. Slieker ${ }^{1,2^{*}}$ (D), Amber A. W. H. van der Heijden³, Giel Nijpels ${ }^{3}$, Petra J. M. Elders ${ }^{3}$, Leen M. 't Hart ${ }^{1,2,4 \dagger}$ \\ and Joline W. J. Beulens ${ }^{1,5+}$
}

\begin{abstract}
Background: Glycemic variation has been suggested to be a risk factor for diabetes-related complications. Previous studies did not address confounding of diabetes duration, number of visits and length of follow-up. Here, we characterize glycemic variability over time and whether its relation to diabetes-related complications and mortality is independent from diabetes- and follow-up duration.

Materials and methods: Individuals with type 2 diabetes $(n=6770)$ from the Hoorn Diabetes Care System cohort were included in this study. The coefficient of variation (CV) was calculated over 5-year sliding intervals. People divided in quintiles based on their CV. Cox proportional hazard models were used to investigate the role of glycemic $\mathrm{CV}$ as risk factor in diabetes-related complications and mortality.

Results: The coefficient of variation of glucose (FG-CV) increased with time, in contrast to HbA1c (HbA1c-CV). People with a high FG-CV were those with an early age of diabetes onset $\left(\triangle_{\mathrm{Q} 5-\mathrm{Q} 1}=-2.39\right.$ years), a higher BMI $\left(\Delta_{\mathrm{Q} 5-}\right.$ $\left.\mathrm{Q}_{1}=+0.92 \mathrm{~kg} / \mathrm{m}^{2}\right)$, an unfavorable lipid profile, i.e. lower levels of $\mathrm{HDL}-\mathrm{C}\left(\Delta_{\mathrm{Q} 5-\mathrm{Q} 1}=-0.06 \mathrm{mmol} / \mathrm{mol}\right)$ and higher triglycerides $\left(\Delta_{\mathrm{Q} 5-\mathrm{Q} 1}=+1.20 \mathrm{mmol} / \mathrm{mol}\right)$. People with the highest $\mathrm{FG}-\mathrm{CV}$ in the first 5-year interval showed an increased risk of insulin initiation, retinopathy, macrovascular complications and mortality independent of mean glycemia, classical risk factors and medication use. For $\mathrm{HbA1c}$, the associations were weaker and less consistent.

Conclusions: Individuals with a higher FG-CV have an unfavorable metabolic profile and have an increased risk of developing micro- and macrovascular complications and mortality. The association of HbA1c-CV with metabolic outcomes and complications was less consistent in comparison to FG-CV.
\end{abstract}

Keywords: Complications, Glycemia, Type 2 diabetes, Variability

\section{Background}

People with type 2 diabetes have a 2-4 times increased risk to develop macrovascular complications $[1,2]$. Therefore, an important challenge for diabetes-related

*Correspondence: r.slieker@amsterdamumc.nl

${ }^{\dagger}$ Leen M 't Hart and Joline W. J. Beulens contributed equally to this work

${ }^{1}$ Department of Epidemiology and Biostatistics, Amsterdam Public

Health Institute, Amsterdam UMC, Location VUMC, De Boelelaan 1089a,

1081 HV Amsterdam, The Netherlands

Full list of author information is available at the end of the article micro- and macrovascular complications is to identify new risk factors.

Over the past years, an increasing number of studies have investigated visit-to-visit glycemic variability as risk factor for diabetes-related complications and its underlying mechanism [3-17]. These studies used a measure of variation of fasting glucose or HbAlc over the follow-up duration to assess glycemic variability and showed that a high glycemic variability is a risk factor for micro- and macrovascular complications and mortality independent of their respective means [3-15]. This increased risk could be explained

(c) The Author(s) 2019. This article is licensed under a Creative Commons Attribution 4.0 International License, which permits use, sharing, adaptation, distribution and reproduction in any medium or format, as long as you give appropriate credit to the original author(s) and the source, provide a link to the Creative Commons licence, and indicate if changes were made. The images or other third party material in this article are included in the article's Creative Commons licence, unless indicated otherwise in a credit line to the material. If material is not included in the article's Creative Commons licence and your intended use is not permitted by statutory regulation or exceeds the permitted use, you will need to obtain permission directly from the copyright holder. To view a copy of this licence, visit http://creativeco mmons.org/licenses/by/4.0/. The Creative Commons Public Domain Dedication waiver (http://creativecommons.org/publicdomain/ zero/1.0/) applies to the data made available in this article, unless otherwise stated in a credit line to the data. 
by associated cardiometabolic risk factors and even cardiac structure [18, 19], but studiescharacterizing the relation between glycemic variability and anthropomorphic, metabolic and lifestyle factors are sparse. Only Noyes et al. investigated this relation and showed a high glycemic variability, defined as the $\mathrm{CV}$ and standard deviation, occurred more often in more intensively treated individuals with a low mean HbA1c [20]. Moreover, a high $\mathrm{HbA1c}$ variability was associated with male sex, a younger age, a lower HDL-C and a higher BMI [20].

Thus far, studies investigating variability in relation to complications [3-13] or individuals' characteristics have mostly used the same approach of calculating variability over the total follow-up time in which people differed in their time since diagnosis, follow-up time, number of visits and the distribution of visits across the follow-up [20]. This approach has several limitations. Individuals with the highest variability are more likely to have a longer time since diagnosis [21, 22]. Also, people with more visits, a higher frequency of visits, and a long follow-up are more likely to have a high variability. More visits and higher frequency in a short period of time are most likely driven by poor glycemic control leading to an increased glycemic variability. This will therefore confound the association and as such the interpretation between glycemic variability and diabetes-related complications. Finally, by combining all follow-up measurements, information is lost about the changes in variability over time.

By investigating the glycemic variability across fixed time intervals of follow-up, aligned with time since diagnosis, with equal time between measurements, one could overcome these confounding factors. We here investigate the relation of glycemic variability over time with clinical characteristics, medication usage and micro- and macrovascular complications and mortality in the Hoorn Diabetes Care System (DCS) cohort, a large prospective cohort of people with type 2 diabetes with annual repeated measures of risk factors and complications.

\section{Methods}

\section{Study population}

People from the Hoorn DCS cohort were included, which is an open prospective cohort of people with type 2 diabetes from the northwest part of the Netherlands [23]. People were diagnosed with type 2 diabetes when they had fasting glucose level $\geq 7 \mathrm{mmol} / \mathrm{l}$ on two separate days, or fasting glucose level $\geq 7 \mathrm{mmol} / \mathrm{l}$ with hyperglycemic symptoms or any glucose level $\geq 11 \mathrm{mmol} / \mathrm{l}$ [24]. As part of routine care, individuals visit the DCS once a year for diabetes monitoring [23].

\section{Selection}

Data of 12,869 individuals (Data freeze 1998-2015) were used for analysis (Additional file 1: Figure S1A). Individuals were excluded when they only had visits within 6 months after diagnosis $(\mathrm{N}=1082)$, a follow-up shorter than 5 years $(\mathrm{N}=4775)$, an age of diabetes onset below 35 years to exclude those with type 1 diabetes $(\mathrm{N}=229)$ or those with missing values $(\mathrm{N}=3)$. In total, 6780 individuals were included for analysis (Additional file 1: Figure S1A).

\section{Clinical characteristics}

Smoking status was self-reported. Systolic and diastolic blood pressure were measured twice at each visit $3 \mathrm{~min}$ apart with the average used for analysis [23]. Current medication use was registered based on the dispensing labels of the medication. All laboratory measurements were measured in a fasted state. All HbAlc measurements were performed using the turbidimetric inhibition immunoassay for hemolyzed whole EDTA blood (Cobas c501, Roche Diagnostics, Mannheim, Germany, run CV 1.6\%) [23], fasting glucose levels were measured in fluorinated plasma using hexokinase with the UV test (Cobas c501, Roche Diagnostics, run CV 1.3\%) [23]. Levels of triglycerides $(\mathrm{mmol} / \mathrm{L})$, total cholesterol $(\mathrm{mmol} / \mathrm{L}), \mathrm{HDL}-\mathrm{C}$ $(\mathrm{mmol} / \mathrm{L})$ and serum creatinine levels were measured enzymatically (Cobas c501, Roche Diagnostics). LDL-C levels $(\mathrm{mmol} / \mathrm{L})$ were derived according from cholesterol- and triglycerides levels [25].

\section{Glycemic variability}

Visits between diagnosis and the first 6 months after diagnosis date were excluded, to reduce the effect on variability of the initial treatment. Glycemic variability was calculated over 5-year intervals, with five measurements 1 year apart (Additional file 1: Figure S1b). Variability in FG and HbA1c for the first 20 intervals was calculated as the standard deviation, median absolute deviation and the coefficient of variation (CV). Five-year intervals were aligned based on time since diagnosis, i.e. the first interval represents $1-5$ years after diagnosis. Not all individuals were in all intervals, where individuals were allowed to be in a subset of the intervals. Only intervals $1-20$ with $\geq 200$ individuals were considered (Interval 1-20, Additional file 2: Table S1). Individuals within an interval were split into quintiles based on their CV $(1=$ low $\mathrm{CV}$, $5=$ high $\mathrm{CV}$ ).

\section{Progression outcomes}

Insulin initiation was defined as treatment with insulin for two subsequent years or the requirement to initiate insulin treatment but without actual treatment. Insulin 
requirement was defined as $\mathrm{HbA1c}$ levels over $>8.5 \%$ or $69 \mathrm{mmol} / \mathrm{mol}$ for two subsequent years while treated with two or more oral glucose-lowering drugs.

Retinopathy state was based on annual fundus photography of both eyes and graded according to the EURODIAB classification score. Time to referable retinopathy was defined as the time between diagnosis and the first occurrence of stage 2: moderate non-proliferative retinopathy or higher [23].

Annual estimated glomerular filtration rate (eGFR) was calculated using the CKD-EPI formula for people of European descent [26]. Based on CKD-EPI eGFR, eGFR stages were defined as: (1) normal or high ( $\geq 90 \mathrm{~mL} /$ $\mathrm{min} / 1.73 \mathrm{~m}^{2}$ ), (2) mildly decreased (60-89), (3a) mildly to moderately decreased (45-59), (3b) moderately to severely decreased (30-44), (4) severely decreased $(15-29)$ and $(5$. kidney failure $(<15)$ [27]. The first date at which stage 4 or an eGFR $<29 \mathrm{~mL} / \mathrm{min} / 1.73 \mathrm{~m}^{2}$ was reached was used as the eGFR endpoint [27].

Stages of chronic kidney disease (CKD) were defined as previously described [27, 28]. Endpoint was the first date at CKD stage 2 or 3, which are people with the following combination:

- eGFR stage $1 / 2$ and albuminuria $>30 \mathrm{mg} / \mathrm{mmol}$

- eGFR stage 3a and albuminuria 3-30 mg/mmol

- eGFR stage $3 \mathrm{~b}$ and albuminuria $<3 \mathrm{mg} / \mathrm{mmol}$

- eGFR stage $4 /$ stage 5

Urinary albumin was measured turbidimetrically (Cobas c501, Roche Diagnostics) and creatinine levels enzymatically (Cobas c501, Roche Diagnostics) as described elsewhere [23]. Micro- and microalbuminuria were defined as the first visit at which the urinary albumin/creatinine ratio was over $2.5 \mathrm{mg} /$ $\mathrm{mmol}$ (male) $/ 3.5 \mathrm{mg} / \mathrm{mmol}$ (female) and $25 \mathrm{mg} / \mathrm{mmol}$ (male) $/ 35 \mathrm{mg} / \mathrm{mmol}$ (female), respectively.

Onset of macrovascular complications was defined as the time upon the first macrovascular event relative to diagnosis. Macrovascular complications were self-reported, but all self-reported events were verified against electronic medical registrations from the regional hospital and GP [23]. We validated this procedure in a subsample of 453 participants showing a sensitivity of $86 \%$ and a specificity of $90 \%$ [23]. ICD-9 410-449 or ICD-10 I10-I79 were included for macrovascular complications, including ischemic heart disease, diseases of pulmonary circulation, other forms of heart disease, cerebrovascular disease, cerebrovascular disease and diseases of arteries, arterioles, and capillaries. Vital status was checked every 6 months using the National population registry [23].

\section{Statistical analysis}

Associations between glycemic variability and continuous determinants were performed using a linear regression with adjustment for sex, age at diagnosis and mean glucose or HbA1c. Associations between glycemic variability and dichotomous determinants were performed using logistic regression adjusted for the same covariates. A model was run for each of the 20 intervals on each of the determinants. $P$-values were adjusted for multiple testingusing the Bonferroni procedure, with $P$-values $\leq 2.5 \cdot 10^{-3}$ (0.05/20 intervals) considered significant.

Cox proportional hazard models were performed to test glycemic variability as risk factor for diabetes progression. Time to event was defined as the time between diagnosis date and date of first event of micro- and macrovascular complications or death. Only first interval was investigated in Cox $\mathrm{PH}$ models to avoid survival bias and models were stratified by glucose or HbA1c tertiles. The crude model was adjusted for age at diagnosis, mean BMI and sex. The fully adjusted model additionally included mean HDL and triglycerides, first HbA1c, oral glucose lowering drugs use, insulin use and eGFR (eGFR, CKD, micro- and macroalbuminuria models). The lowest quintile of glycemic variability was used as the reference group. A trend test across glycemic variability quintiles was performed by including the median coefficient of variation of fasting glucose (FG-CV) and $\mathrm{HbA1c}$ (HbA1c-CV) of each quintile as continuous variable in the model. Individuals with missing data in the models were removed $(<0.5 \%)$. All analyses were performed with R statistics (version 3.5.1) in combination with the $\mathrm{R}$ packages survival (2.43-3) and ggplot2 (version 3.1.0).

\section{Results}

Clinical characteristics of the participants in the first interval are shown in Table 1 . Time since diagnosis was on average 1.1 years $(\mathrm{IQR}=0.9-1.2$ years, Table 1$)$. Both the coefficient of variation of fasting glucose (FG-CV) and HbA1c (HbA1c-CV) showed the lowest correlation with the mean fasting glucose and HbA1c, respectively $\left(r_{\mathrm{FG}}=0.42\right.$ and $\left.\mathrm{r}_{\mathrm{HbAlc}}=0.49\right)$, in contrast to the standard deviation and the median absolute deviation and where therefore used to reduce the effect of mean glycemia.

At baseline, individuals with a high FG-CV had on average a significantly higher $\mathrm{BMI}(\mathrm{Q} 1=29.1 \mathrm{vs} \mathrm{Q} 5=30.8 \mathrm{~kg} /$ $\left.\mathrm{m}^{2}\right)$, higher fasting glucose $(\mathrm{Q} 1=7.1, \mathrm{Q} 5=8.9 \mathrm{mmol} / \mathrm{L})$ and higher triglycerides $(\mathrm{Q} 1=1.7, \mathrm{Q} 5=2.2 \mathrm{mmol} / \mathrm{L}$, Table 1). The proportion current smokers was higher in the highest quintile group ( $\mathrm{Q} 5=26.0 \%$ vs $\mathrm{Q} 1=16.2 \%$ ). Individuals with a high FG-CV were more often on metformin $(\mathrm{Q} 1=48 \%, \mathrm{Q} 5=58 \%)$, sulphonylureas (SU, $\mathrm{Q} 1=14 \%, \mathrm{Q} 5=42 \%)$ and insulin $(\mathrm{Q} 1=2 \%, \mathrm{Q} 5=12 \%$, 


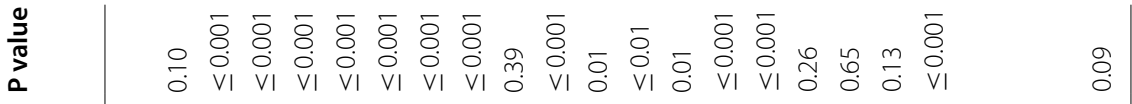

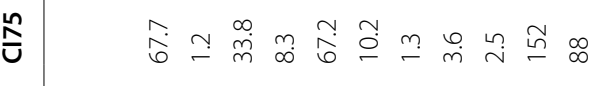

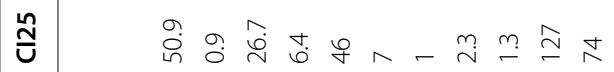

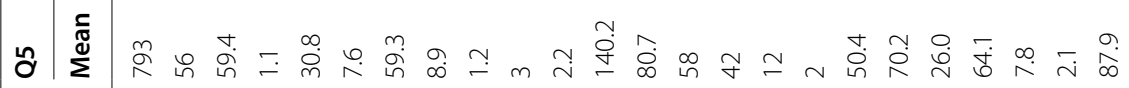

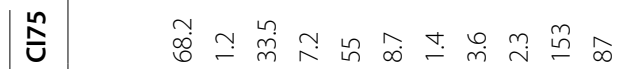

ปี

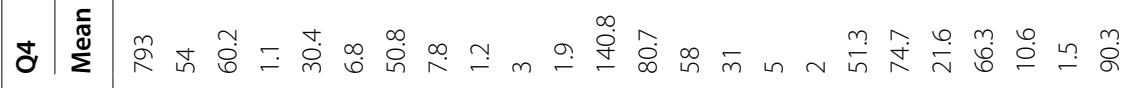

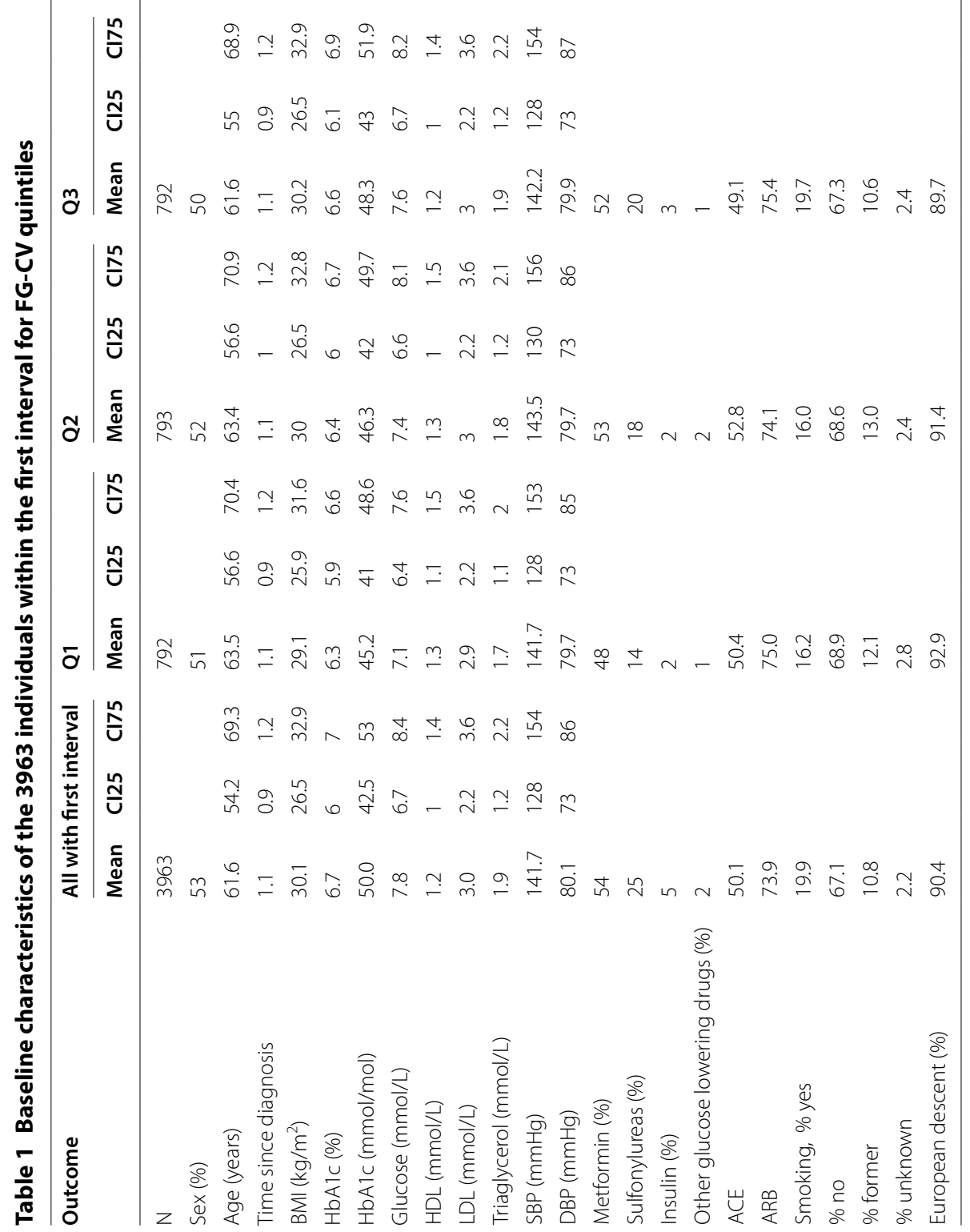


Table 1). The characteristics for $\mathrm{HbA1c}-\mathrm{CV}$ quintiles resembled those of FG-CV (Additional file 3: Table S2).

\section{FG-CV increases over time in contrast to HbA1c-CV}

FG-CV increased in almost a linear fashion across the 5-year intervals (Fig. 1a, Additional file 4: Figure S2a), while $\mathrm{HbA1c}-\mathrm{CV}$ remained largely stable over time (Fig. 1b-e). The correlation between HbA1c-CV and FG-CV also declined with time (Additional file 4: Figure S2b). In the first interval, individuals were often in the same interval for FG-CV and HbA1c-CV (Additional file 4: Figure S2d), although a substantial number was also in neighboring quintiles.

Between the lowest and the highest FG-CV quintiles, the differences in fasting glucose variability were clearly visible (Fig. 1f). Individuals with low or high FG-CV and $\mathrm{HbA1c-CV}$ largely remained in the same quintile across their follow-up (Fig. 1g and Additional file 4: Figure S2c). For FG-CV, $74.4 \%$ had at least $50 \%$ of their intervals in one quintile and $72.0 \%$ had at least $75 \%$ of their intervals in two adjacent quintiles.
High FG-CV associates with higher triglycerides and BMI and lower HDL-C levels and an earlier disease onset The profile of people with a high FG-CV or HbA1C$\mathrm{CV}$ was largely similar. For sex, no consistent difference was observed in FG-CV or HbA1c-CV $\left(P_{\text {bonf }}>0.05\right.$, Fig. 2a, Additional file 5: Table S3, Additional file 6: Table S4). Individuals with a high FG-CV and HbA1c$C V$ were those with an early diagnosis age $\left(\mathrm{Q}_{2}=0.26\right.$, $\mathrm{Q}_{5}=-2.39$ years; Additional file 5: Table S3, Fig. 2b). A high FG-CV and $\mathrm{HbA1c}-\mathrm{CV}$ was associated with a higher $B M I$ in the first interval $\left(Q_{2}=0.76, Q_{5}=0.92 \mathrm{~kg} /\right.$ $\mathrm{m}^{2}$; Fig. 2b, Additional file 5: Table S3). The difference in BMI persisted over time for both FG-CV and HbA1c-CV. Finally, a high FG-CV was associated with lower HDL-C levels $\left(\mathrm{Q}_{2}=-0.01, \mathrm{Q}_{5}=-0.06 \mathrm{mmol} / \mathrm{L}\right.$, Fig. 2b, Additional file 5: Table S3) and a higher level of triglycerides $\left(\mathrm{Q}_{2}=1.02, \mathrm{Q}_{5}=1.07 \mathrm{mmol} / \mathrm{L}\right.$; Fig. 2g, Additional file 5: Table S3). The lower HDL-C levels and higher triglycerides in the highest FG-CV quintile persisted for most intervals (Fig. 2f, g) and resembled the results for HbA1cCV (Additional file 7: Figure S3f, g, Table S4). For total cholesterol (Fig. 2e), blood pressure (Fig. 2h-i), eGFR

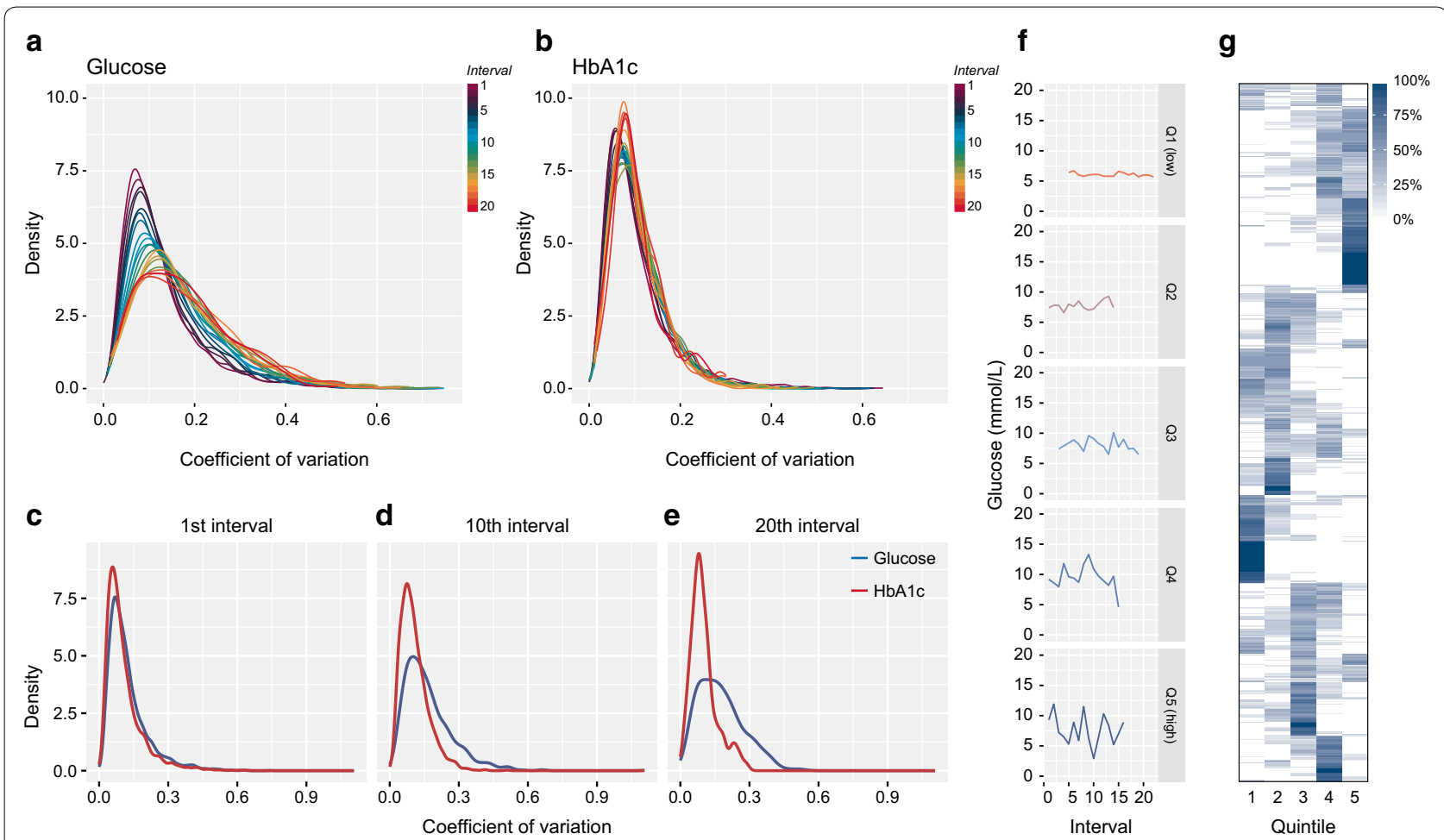

Fig. 1 Glucose- and HbA1c-CV across time. Density plot of glucose-CV (a) and HbA1c (b) across the first 20 intervals. X-axis, level of FG-CV; $y$-axis: smoothed frequency; Colors represent the intervals with purple the 1st interval and red the 20th interval, density plot of the glucose-CV (blue) vs HbA1c-CV (red) in the first interval (c), tenth interval (d) and twentieth interval (e). $\mathbf{f}$ Example of five individuals that remained in the same quintile across all intervals across their follow-up. Each row represents one individual and each column the five quintiles. $\mathbf{g}$ Percentage of intervals compared to total number of individuals that ended up in a certain quintile, i.e. 100\% means all intervals of an individual were assigned to that quintile 

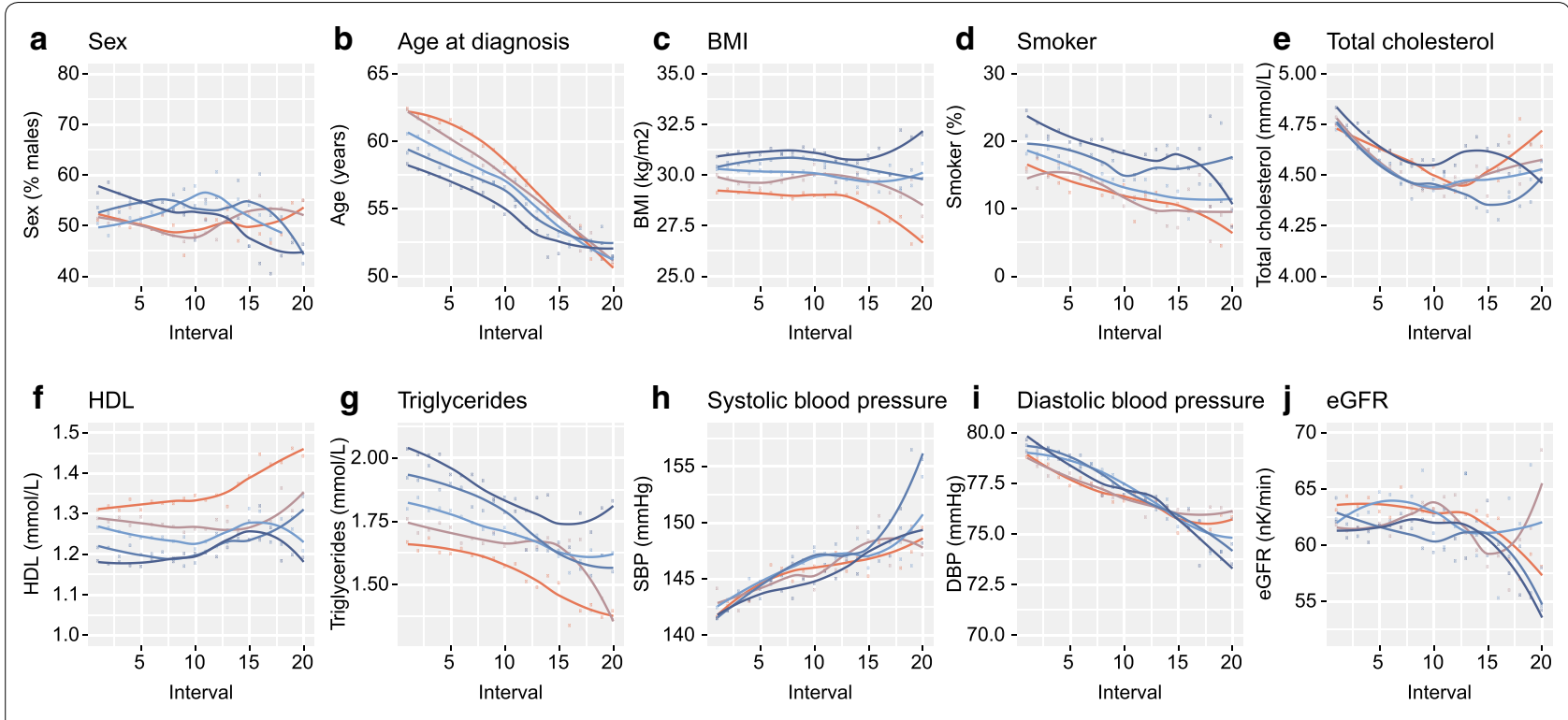

$\leftarrow 1$ (Low variability) $\leftarrow 2 \div 3 \div 4 \div 5$ (High variability)

Fig. 2 Characterization of individuals based on their FG-CV. Unadjusted outcome against the five quintiles with the fifth quintile being the group with the highest FG-CV against a sex (\% males), b age at diagnosis, c BMI, $\mathbf{d} \%$ smokers, e total cholesterol levels, $\mathbf{f}$ HDL-C levels, $\mathbf{g}$ triglycerides levels, $\mathbf{h}$ DBP, $\mathbf{i}$ SBP, $\mathbf{j}$ eGFR. Lines represent loess smoothed levels over 5-year average values of outcomes

(Fig. 2j), and smoking (Fig. 2d) no consistent associations were observed across FG-CV quintiles (Fig. 2e, h-j; Additional file 6: Table S4). A high HbA1c-CV was however, associated with a higher diastolic blood pressure (intervals $1-7,9 ; P_{\text {bonf }} \leq 0.04$, but not SBP (Additional file 6; Table S4, Additional file 7: Figure S3). Of note, limiting the analysis to only the people that were included in the first interval did not change the result (data not shown).

\section{A high FG-CV is associated with more intense treatment and earlier insulin initiation}

Individuals with a high $\mathrm{FG}-\mathrm{CV}$ and $\mathrm{HbA1c}-\mathrm{CV}$ were different in how they were treated. A low proportion of individuals were drug-naïve in the highest FG-CV (2.5\%) and $\mathrm{HbA} 1 \mathrm{c}-\mathrm{CV}(2.8 \%)$ versus the lowest quintile $(33.0 \%$ and $34.6 \%$, Fig. 3a). The proportion of individuals on dual therapy with both metformin and SU quickly decreased with time in the highest FG-CV and HbA1c-CV groups (38.5\% and $42.4 \%$ to $9.8 \%$ and $19.5 \%$ ), while in the lowest quintile the number of individuals treated with both metformin and SU increased (15.9\% and $14.6 \%$ to $45 \%$ and 30\%, Fig. 3b-d).

Relative to the lowest FG-CV quintile, those in the highest FG-CV quintile had a significantly higher use of metformin and SU use in the first 11 and nine intervals respectively (Fig. 3f, g, Additional file 8: Table S5). For HbA1c-CV similar observations were seen (Additional file 9: Figure S4).
Insulin use was much higher in the highest quintile versus the lowest quintile (Fig. 3e), across all 20 intervals with an OR of 19.37 (95\% CI 12.86-30.55; $P_{\text {trend }}=4.11 \cdot 10^{-40}$ ) in the first interval and 17.50 (95\% CI 6.05-58.39; $P_{\text {trend }}=2.22 \cdot 10^{-7}$ ) in the 20th interval (Fig. 3h, Additional file 8: Table S5). Individuals with a high FG-CV also went faster on insulin (Fig. 3e) with HRs ranging from $1.00\left(\mathrm{Q}_{2} 95 \% \mathrm{CI} 0.75-1.33 ; P\right.$ value $\left.=0.98\right)$ to $3.33(\mathrm{Q} 5$ 95\% CI 2.61-4.27, $\left.P=8.43 \cdot 10^{-22}\right)$ and $P_{\text {trend }}=3.31 \cdot 10^{-44}$ (Table 2) in the fully adjusted model. For $\mathrm{HbA1c}-\mathrm{CV}$, lower hazards were observed than FG-CV, ranging from $1.52\left(\mathrm{Q}_{2} 95 \% \mathrm{CI} 1.13-2.04, P=5.38 \cdot 10^{-3}\right)$ to $2.45\left(\mathrm{Q}_{5} 95 \%\right.$ CI 1.86-3.22, $P=2.00 \cdot 10^{-10}$ and $P_{\text {trend }}=4.00 \cdot 10^{-11}$ ).

\footnotetext{
A high FG-CV is a risk factor for retinopathy, macrovascular complications and mortality

FG-CV and HbA1c-CV of the first interval (year 1-5 after diagnosis, $\mathrm{N}=3963$ ) were investigated as risk factors for diabetes-related complications during follow-up (median 8-9 years). A high FG-CV in the first interval was associated with proliferative retinopathy independent of mean fasting glucose (Table 2). The two highest quintiles were significant $\left(\mathrm{HR}_{\mathrm{Q} 4}=2.35[1.22-4.51]\right.$, $P=0.01, \mathrm{HR}_{\mathrm{Q} 5}=2.59[1.34-5.01], P=4.56 \cdot 10^{-3}$, Table 2), with the overall trend also significant $\left(P_{\text {trend }}=1.08 \cdot 10^{-3}\right)$. HbA1c-CV in the first interval was not associated with retinopathy $\left(P_{\text {trend }}=0.80\right)$.
} 


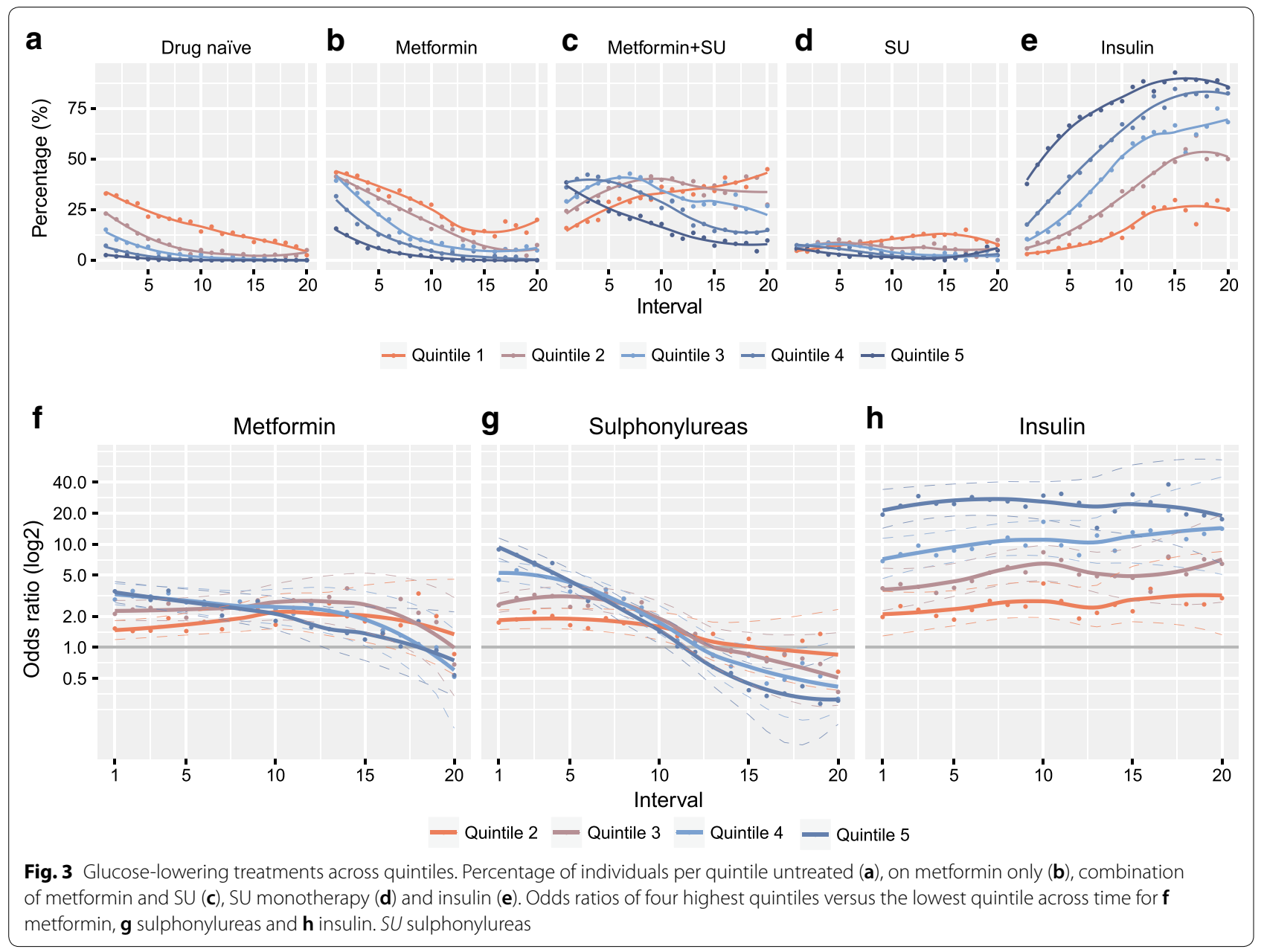

In relation to kidney-related complications, FG-CV was a strong risk factor for microalbuminuria $\quad\left(\mathrm{HR}_{\mathrm{Q} 5}=1.63 \quad[1.36-1.96], \quad P=1.33 \cdot 10^{-7}\right.$, $\left.P_{\text {trend }}=4.23 \cdot 10^{-7}\right)$ and macroalbuminuria $\left(\mathrm{HR}_{\mathrm{Q} 5}=2.89\right.$ $[1.82-4.60], P=7.40 \cdot 10^{-6}, P_{\text {trend }}=2.98 \cdot 10^{-7}$ ). The hazards for $\mathrm{HbA1c-CV}$ were generally lower and were only significant for microalbuminuria $\left(\mathrm{HR}_{\mathrm{Q} 5}=1.38\right.$ [1.141.66], $\left.P=7.11 \cdot 10^{-4}, P_{\text {trend }}=1.43 \cdot 10^{-4}\right)$. eGFR stage did not show any consistent results for FG-CV or HbA1c$\mathrm{CV}$. The hazards for CKD stage did increase for FG-CV, but not HbA1c-CV, but were not significant $(P>0.05)$.

Higher FG-CV was associated $\left(P_{\text {trend }}=3.96 \cdot 10^{-3}\right)$ with a higher risk to develop macrovascular complications, with hazard ratios of 1.39 (95\% CI 1.01-1.91, $P=0.04$ ) for the third quintile, 1.43 (95\% CI 1.02-1.94, $P=0.04)$ for the fourth quintile and 1.62 (95\% CI 1.162.26, $P=4.28 \cdot 10^{-3}$ ) for the highest quintile (Table 2). For $\mathrm{HbA1c-CV}$, the effects were largely in the same direction, but only significant in the highest quintile $\left(\mathrm{HR}_{\mathrm{Q} 5}=1.39[1.01-1.91], P=0.05, P_{\text {trend }}=0.04\right)$.
Finally, a higher FG-CV was associated with a higher mortality risk for the three highest quintiles, but only the second $\left(\mathrm{HR}_{\mathrm{Q} 2}=1.34[1.03-1.75], P=0.03\right)$ and the highest quintile $\left(\mathrm{HR}_{\mathrm{Q} 5}=1.69[1.29-2.22], P=1.34 \cdot 10^{-4}\right)$ showed a significantly higher mortality risk $\left(P_{\text {trend }}=1.03 \cdot 10^{-5}\right.$, Table 2). For HbA1c-CV, the highest quintile was significantly associated with an increased risk of mortality $\left(\mathrm{HR}_{\mathrm{Q} 5}=1.38[1.07-1.80], P=0.01, P_{\text {trend }}=6.87 \cdot 10^{-4}\right)$.

\section{Discussion}

We investigated the differences in characteristics of people with low and high glycemic variability in people with type 2 diabetes. FG-CV across intervals increased with time, while $\mathrm{HbA} 1 \mathrm{c}-\mathrm{CV}$ remained largely stable and the correlation between the two decreased. Both a higher FG-CV and HbA1c-CV were characterized by an earlier disease onset, a higher BMI, lower HDL-C and higher triglycerides. Insulin use, and initiation was consistently higher in those with a high CV. Individuals with a high FG-CV in the first 5 years-but to a lesser extent 


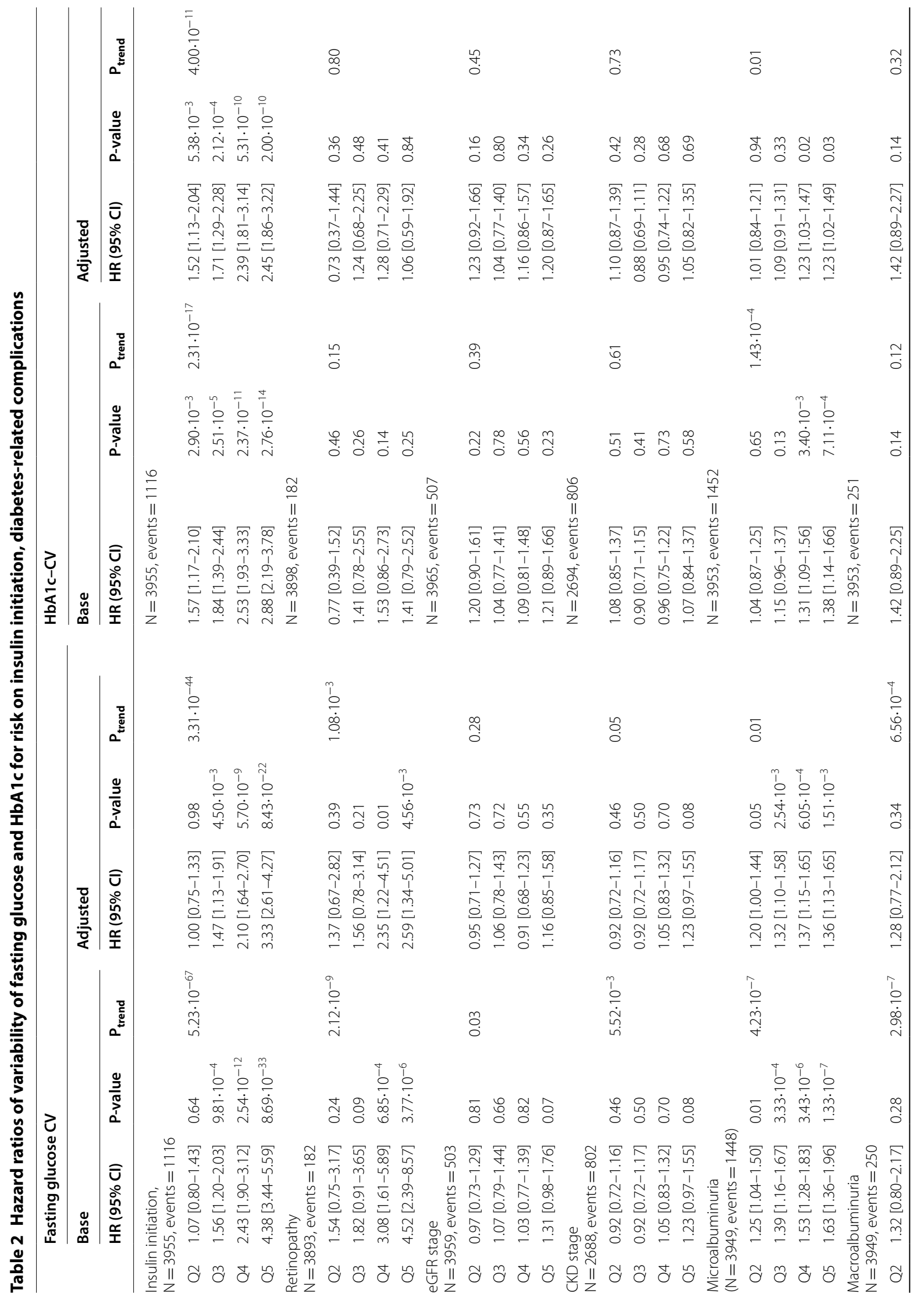




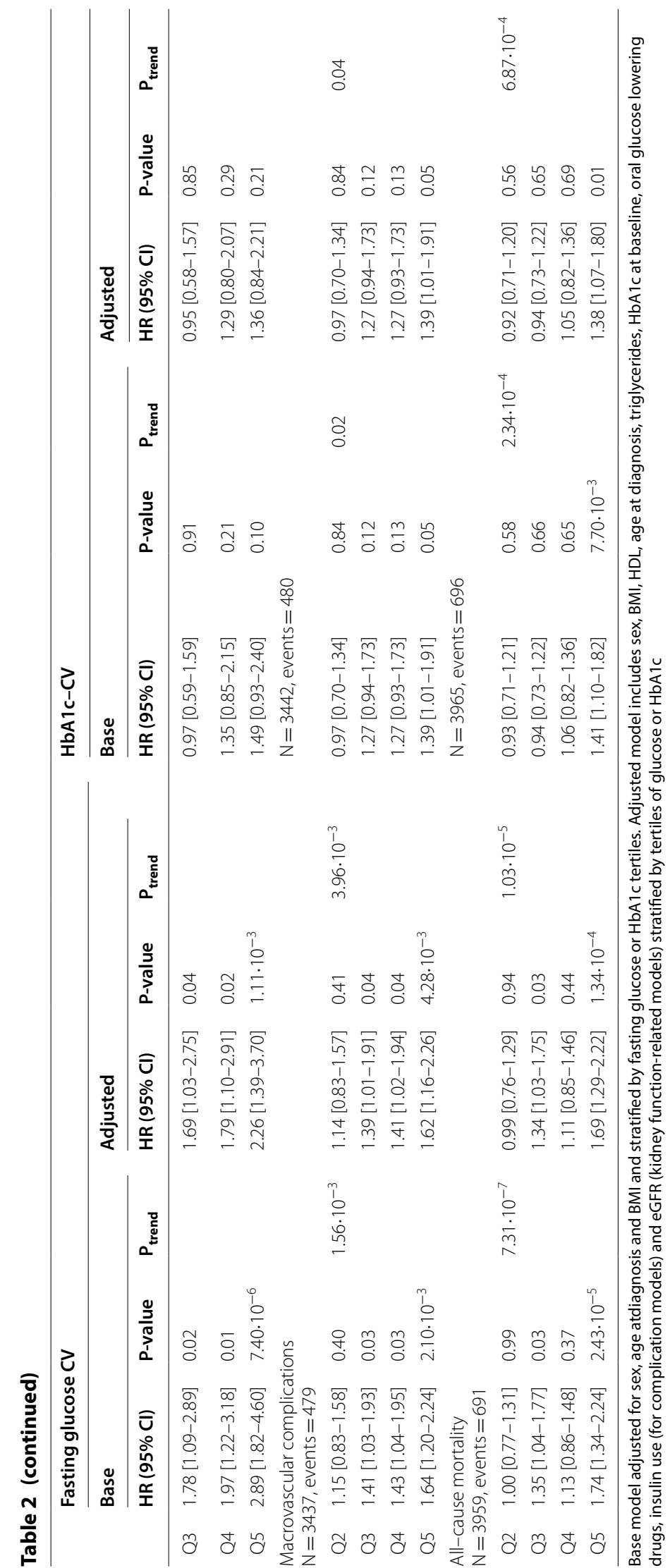


for HbA1c-CV-showed an increased risk to develop retinopathy, macrovascular complications and were at increased risk of mortality, independent of mean FG or HbA1c, time since diagnosis and number of follow-up measurements.

A high HbA1c-CV and FG-CV was associated with an earlier disease onset, suggesting that those in which the disease onset is early in life suffer from a poorer glycemic control compared to those with a late onset [29].

Individuals with a high FG-CV in the first 5-years of their disease were at high risk to initiate insulin compared to those with a low FG-CV. A previous study showed that individuals with higher HbA1c variability were those on a more extensive treatment regimen, compared to those with a low HbA1c variability [20]. People with a high glycemic variability have been shown to be more often insulin deficient, which could be an explanation for their higher risk on insulin initiation [30].

\section{FG-CV over the first 5 years is a risk factor for micro- and macrovascular complications}

FG-CV and to a lesser extent $\mathrm{HbA1c}-\mathrm{CV}$ were risk factors for micro- and macrovascular complications. The number and timing of visits was the same in all study subjects these observations were. This in contrast to many previous studies that have looked at FG-CV and HbA1c as risk factors for diabetes-related complications. For microvascular complications, an increased risk was observed for retinopathy with increasing FG-CV. In two cross-sectional studies, contradicting results were observed between FG-CV and retinopathy outcome, but this may be due to the heterogeneity of the studies in terms of number of follow-up measurements and time since diagnosis $[3,31]$. A meta-analysis on $\mathrm{HbA} 1 \mathrm{c}$ variability did not find a relation with retinopathy, although this was based on only two studies and not based on variability of fasting glucose [32].

A high FG-CV in the first interval was associated with an increased risk of incident macrovascular complications, much stronger than HbA1c-CV. An increased risk for macrovascular complications has been observed in previous studies $[6,17]$, for example, FG-CV has been shown to be a risk factor for ischemic stroke in Taiwanese [33], but also with stroke and myocardial infarction in a German population [8]. Although we did see a relation in the fully adjusted models for incident CVD, a crosssectional study did not find a relation for $\mathrm{HbA1c}-\mathrm{CV}$ with CVD [34].

In terms of kidney-related complications, FG-CV and to a lesser extent $\mathrm{HbA1c}-\mathrm{CV}$ were risk factors for microand macroalbuminuria. For eGFR-stages no consistent associations were found for both FG-CV and $\mathrm{HbAlc}-\mathrm{CV}$. In the CKD-stages a modest effect was seen, but this is likely driven by the stronger effects of the albuminuria. In a previous study, FG-SD-but not Hba1c-SD-was associated with microalbumuria [16]. Other studies have shown that the glycemic variability measures $\mathrm{HbA1c}-\mathrm{CV}$ and SD were risk factors for chronic kidney disease with modest effect sizes $[4,35,36]$. In a meta-analysis of these studies HbA1c-SD was also identified as a risk factor for CKD [37]. Explanations for lack of association between $\mathrm{CV}$ and kidney-related complications are the complexity of the outcome, including differences in genetic background and medication use. In addition, earlier studies may have been confounded by diabetes duration or follow-up time. Since age is a very important predictor of kidney function decline, accounting for such age-related factors may also explain the lack of association in our study.

Finally, we observed FG-CV and to a lesser extend HbA1c-CV to be a risk factor for mortality. In previous studies similar mortality risks were observed $[6,9,16$, 38]. HbA1c-CV was only associated with mortality in the adjusted model, but not in the crude model.

The different distribution of individuals over quintiles in FG-CV vs HbA1c-CV and the subsequently different results for both measures, suggest that $\mathrm{HbA1C}-\mathrm{CV}$ and FG-CV are not mutually exclusive. Fasting glucose levels reflect an individual's ability to regulate glucose levels in absence of dietary glucose intake and is thereforeindependent of the dietary glucose intake itself. In previous studies it has been shown that postprandial glucose levels correlate better to HbA1c. Moreover, our results suggest that levels of variability of fasting glucose are of added value to solely measuring HbA1c. After all, a high FG-CV in the first 5 years after diagnosis was already indicative of a higher risk to develop retinopathy, macrovascular complications and mortality. Future studies should explore whether measures of glycemic variability over time can improve prediction of vascular complications in people with type 2 diabetes.

The strengths of our study include its large sample size with long follow-up duration, a deeply phenotyped cohort with highly standardized annual measurements, which allow us to compare the FG-CV with various outcomes across $>20$ years of disease duration and follow-up. Another strength of our study is that the time between follow-up measurements is relatively constant with individuals visiting DCS once a year rather than most other studies where the frequency of sampling over years may vary considerably. A final strength of our study is that we aligned the visits based on time since diagnosis and the number of visits/measurements per interval.

A limitation of our study is the number of individuals varied across intervals. As such the individuals with a longer follow-up were generally those with an earlier 
disease onset. A second limitation is that intervals were aligned based on the date of diagnosis, while ideally one would use the date of disease onset. A final limitation is that the data is limited to visit-to-visit, while ideally one would also investigate the relation to continuous glucose monitoring.

FG-CV increased with time, while HbA1c-CV did not. This suggests that FG-CV adds information to HbA1c levels alone, particularly in people with a longer diabetes duration. A high FG-CV was associated with poorer health profile compared to those with low FG-CV, characterized by an earlier age of disease onset, a higher BMI, low HDL-C and higher triglycerides levels and individuals were more often current smokers. Individuals with a high FG-CV had a high risk for insulin initiation across the entire follow-up most likely related to more intensive treatment. Finally, FG-CV was a risk factor for retinopathy, macrovascular compilations and mortality independent of time since diagnosis, follow-up duration and mean glucose concentrations.

\section{Conclusion}

Our results show that fasting glucose variability in the first 5 years-independent of mean glycaemia-is informative of current health status but may also be predictive of faster progression towards insulin and higher risk on complications. For $\mathrm{HbA1c}$, the associations were weaker and less consistent. Our results suggest that FG-CV could serve as an important indicator for diabetes progression, in addition to classical measures such as HbA1c.

\section{Supplementary information}

Supplementary information accompanies this paper at https://doi. org/10.1186/s12933-019-0975-1.

Additional file 1: Figure S1. Study setup. a) Flowchart of the individuals excluded from the analyses. b) Schematic representation of the calculation of variability in 5-year intervals.

Additional file 2: Table S1. Number of individuals per interval.

Additional file 3: Table S2. Characteristics of individuals across $\mathrm{HbA1C}-\mathrm{CV}$ quintiles in the first interval.

Additional file 4: Figure S2. Comparison of FG-CV and HbA1c-CV across intervals. a) Median FG-CV across intervals. Line represents smoothed medians. Light blue, fasting glucose, dark blue HbA1c. b) Spearman correlation between $\mathrm{FG}-\mathrm{CV}$ and $\mathrm{HbA1c}-\mathrm{CV}$ across intervals. Line represents a linear regression line. c) Percentage of intervals compared to total number of individuals that ended up in a certain quintile, i.e. 100\% means all intervals of an individual were assigned to that quintile. d) Overlap between $\mathrm{FG}-\mathrm{CV}$ quintiles and $\mathrm{HbA1c-CV}$ quintiles.

Additional file 5: Figure S3. Characterization of individuals based on their $\mathrm{HbA} 1 \mathrm{c}-\mathrm{CV}$. Unadjusted outcome against the five quintiles with the fifth quintile being the group with the highest FG-CV against (a) sex (\% males) (b) age at diagnosis (c) BMI (d) \% smokers (e) total cholesterol levels (f) HDL-C levels (g) triglycerides levels (h) DBP (i) SBP (j) eGFR. Lines represent loess smoothed levels over 5-year average values of outcomes.

Additional file 6: Table S3. Unadjusted and adjusted effect sizes of models with $\mathrm{FG}-\mathrm{CV}$ for each quintile.

Additional file 7: Table S4. Unadjusted and adjusted effect sizes of models with $\mathrm{HbA} 1 \mathrm{c}-\mathrm{CV}$ for each quintile.

Additional file 8: Table S5. Odds ratios of medication use (metformin, SU, insulin) across intervals. The lowest quintile is used as the reference group.

Additional file 9: Figure S4. Glucose-lowering treatments across $\mathrm{HbA1C}$ CV quintiles. a-e Percentage of individuals per quintile untreated (a), on metformin only (b), combination of metformin and SU (c), SU monotherapy (d) and insulin (e). $f-h$ Odds ratios of the four highest quintiles versus the lowest quintile across time for ( $f$ ) metformin (g) sulphonylureas and (h) insulin. Abbreviations: SU, sulphonylureas.

\section{Abbreviations}

CKD: chronic kidney disease; CV: coefficient of variation; DBP: diastolic blood pressure; eGFR: estimated Glomerular Filtration Rate; FG: fasting glucose; GV: glycemic variability; HR: hazard ratio; Q: quintile; SBP: systolic blood pressure; SU: sulphonylurea derivatives.

\section{Acknowledgements}

The authors thank the people in the Hoorn DCS cohort for participation.

\section{Authors' contributions}

RCS, JWJB, LMtH designed the study. RCS analyzed the data and wrote the draft manuscript. AAWAvdH, GN, JWJB, LMtH, PJE acquired the data from the Hoorn DCS cohort. All authors critically read and revised the manuscript. RCS is the guarantor of this work and, as such, had full access to all the data in the study and takes responsibility for the integrity of the data and the accuracy of the data analysis. All authors read and approved the final manuscript.

\section{Funding}

RCS, LMTH, JWJB received support from the Innovative Medicines Initiative 2 Joint Undertaking under grant agreement No. 115881 (RHAPSODY). This Joint Undertaking receives support from the European Union's Horizon 2020 research and innovation programme and EFPIA. This work is supported by the Swiss State Secretariat for Education, Research and Innovation (SERI) under Contract Number 16.0097-2. The opinions expressed and arguments employed herein do not necessarily reflect the official views of these funding bodies. Support for this research was provided by the Dutch Diabetes Research Foundation (Grant No. 2014.00.1753).

\section{Availability of data and materials} Not applicable.

\section{Ethics approval and consent to participate}

The study has been approved by the Ethical Review Committee of the VU University Medical Center, Amsterdam. Individuals were informed about the use of their anonymized data and were offered an opt-out. A subset of the cohort of 5630 individuals gave consent for linkage to retrieve macrovascular complications.

\section{Consent for publication}

Not applicable.

\section{Competing interests}

The authors declare that they no competing interests.

\section{Author details}

${ }^{1}$ Department of Epidemiology and Biostatistics, Amsterdam Public Health Institute, Amsterdam UMC, Location VUMC, De Boelelaan 1089a, 1081 HV Amsterdam, The Netherlands. ${ }^{2}$ Department of Cell and Chemical Biology, Leiden University Medical Center, Leiden, The Netherlands. ${ }^{3}$ Department of General Practice and Elderly Care Medicine, Amsterdam Public Health Institute, Amsterdam UMC, Location VUmc, Amsterdam, The Netherlands. ${ }^{4}$ Molecular Epidemiology Section, Department of Biomedical Data Sciences, 
Leiden University Medical Center, Leiden, The Netherlands. ${ }^{5}$ Julius Center for Health Sciences and Primary Care, University Medical Center Utrecht, Utrecht, The Netherlands.

Received: 19 October 2019 Accepted: 3 December 2019 Published online: 12 December 2019

\section{References}

1. Gregg EW, Li Y, Wang J, Rios Burrows N, Ali MK, Rolka D, Williams DE, Geiss L. Changes in diabetes-related complications in the United States, 1990-2010. N Engl J Med. 2014;370(16):1514-23.

2. Collaboration Emerging Risk Factors. Diabetes mellitus, fasting blood glucose concentration, and risk of vascular disease: a collaborative metaanalysis of 102 prospective studies. Lancet. 2010;375(9733):2215-22.

3. Gimeno-Orna JA, Castro-Alonso FJ, Boned-Juliani B, Lou-Arnal LM. Fasting plasma glucose variability as a risk factor of retinopathy in type 2 diabetic patients. J Diabetes Complications. 2003;17(2):78-81.

4. Luk AO, Ma RC, Lau ES, Yang X, Lau WW, Yu LW, Chow FC, Chan JC, So WY. Risk association of $\mathrm{HbA1c}$ variability with chronic kidney disease and cardiovascular disease in type 2 diabetes: prospective analysis of the Hong Kong Diabetes Registry. Diabetes Metabolism Res Rev. 2013;29(5):384-90.

5. Bouchi R, Babazono T, Mugishima M, Yoshida N, Nyumura I, Toya K, Hayashi T, Hanai K, Tanaka N, Ishii A, et al. Fluctuations in HbA1c are associated with a higher incidence of cardiovascular disease in Japanese patients with type 2 diabetes. J Diabetes Investig. 2012;3(2):148-55.

6. Hirakawa Y, Arima H, Zoungas S, Ninomiya T, Cooper M, Hamet P. Impact of visit-to-visit glycemic variability on the risks of macrovascular and microvascular events and all-cause mortality in type 2 diabetes: the ADVANCE trial. Diabetes Care. 2014:37:2359-65.

7. Takao T, Matsuyama Y, Suka M, Yanagisawa H, Iwamoto Y. The combined effect of visit-to-visit variability in $\mathrm{HbA1c}$ and systolic blood pressure on the incidence of cardiovascular events in patients with type 2 diabetes. BMJ Open Diabetes Res Care. 2015:3(1):e000129.

8. Bonke FC, Donnachie E, Schneider A, Mehring M. Association of the average rate of change in $\mathrm{HbA1c}$ with severe adverse events: a longitudinal evaluation of audit data from the Bavarian Disease Management Program for patients with type 2 diabetes mellitus. Diabetologia. 2016;59(2):286-93.

9. Prentice JC, Pizer SD, Conlin PR. Identifying the independent effect of $\mathrm{HbA1c}$ variability on adverse health outcomes in patients with Type 2 diabetes. Diabetic Med. 2016;33(12):1640-8.

10. Wan EYF, Fung CSC, Wong CKH, Chin WY, Lam CLK. Association of hemoglobin A1c levels with cardiovascular disease and mortality in chinese patients with diabetes. J Am Coll Cardiol. 2016:67(4):456-8.

11. Zinman B, Marso SP, Poulter NR, Emerson SS, Pieber TR, Pratley RE, Lange M, Brown-Frandsen K, Moses A, Ocampo Francisco AM, et al. Day-today fasting glycaemic variability in DEVOTE: associations with severe hypoglycaemia and cardiovascular outcomes (DEVOTE 2). Diabetologia. 2018:61(1):48-57.

12. Muggeo M, Verlato G, Bonora E, Ciani F, Moghetti P, Eastman R, Crepaldi G, De Marco R. Long-term instability of fasting plasma glucose predicts mortality in elderly NIDDM patients: the Verona Diabetes Study. Diabetologia. 1995;38(6):672-9.

13. Muggeo M, Verlato G, Bonora E, Zoppini G, Corbellini M, De Marco R. Long-term instability of fasting plasma glucose, a novel predictor of cardiovascular mortality in elderly patients with non-insulin-dependent diabetes mellitus. Circulation. 1997:96(6):1750-4.

14. Orsi E, Solini A, Bonora E, Fondelli C, Trevisan R, Vedovato M, Cavalot F, Gruden G, Morano S, Nicolucci A. Haemoglobin A1c variability is a strong, independent predictor of all-cause mortality in patients with type 2 diabetes. Diabetes Obes Metab. 2018;20:1885-93.

15. Lin CC, Chen CC, Chen FN, Li Cl, Liu CS, Lin WY. Risks of diabetic nephropathy with variation in hemoglobin A1C and fasting plasma glucose. Am J Med. 2013;126:e1-10.

16. Cardoso CRL, Leite NC, Moram CBM, Salles GF. Long-term visit-to-visit glycemic variability as predictor of micro- and macrovascular complications in patients with type 2 diabetes: the Rio de Janeiro Type 2 Diabetes Cohort Study. Cardiovasc Diabetol. 2018;17(1):33.
17. Zhou JJ, Schwenke DC, Bahn G. Reaven PJDc: Glycemic variation and cardiovascular risk in the veterans affairs diabetes trial. 2018;41(10):2187-94.

18. Tang $X$, Zhong J, Zhang H, Luo Y, Liu X, Peng L, Zhang Y, Qian X, Jiang B, Liu J, et al. Visit-to-visit fasting plasma glucose variability is an important risk factor for long-term changes in left cardiac structure and function in patients with type 2 diabetes. Cardiovasc Diabetol. 2019;18(1):50.

19. Matsutani D, Sakamoto M, luchi H, Minato S, Suzuki H, Kayama Y, Takeda $\mathrm{N}$, Horiuchi R, Utsunomiya K. Glycemic variability in continuous glucose monitoring is inversely associated with baroreflex sensitivity in type 2 diabetes: a preliminary report. Cardiovasc Diabetol. 2018;17(1):36

20. Noyes J, Soto-Pedre E, Donnelly L, Pearson E. Characteristics of people with high visit-to-visit glycaemic variability in Type 2 diabetes. Diabetic Med. 2017;35(2):262-9.

21. Yang CP, Li Cl, Liu CS, Lin WY, Hwang KL, Yang SY, Li TC, Lin CC. Variability of fasting plasma glucose increased risks of diabetic polyneuropathy in T2DM. Neurology. 2017:88(10):944-51.

22. Low S, Lim SC, Yeoh LY, Liu YL, Liu JJ, Fun S, Su C, Zhang X, Subramaniam T, Sum CF. Effect of long-term glycemic variability on estimated glomerular filtration rate decline among patients with type 2 diabetes mellitus: Insights from the Diabetic Nephropathy Cohort in Singapore. J Diabetes. 2016;9(10):908-19.

23. van der Heijden AA, Rauh SP, Dekker JM, Beulens JW, Elders P, M't Hart L, Rutters F, Leeuwen N, Nijpels G. The Hoorn Diabetes Care System (DCS) cohort. A prospective cohort of persons with type 2 diabetes treated in primary care in the Netherlands. BMJ Open. 2017;7(5):e015599.

24. Rutten G, De Grauw W, Nijpels G, Houweling S, Van de Laar F, Bilo H, Holleman F, Burgers J, Wiersma T, Janssen P. NHG-Guideline Diabetes mellitus type 2 (third revision). Huisarts Wetenschap. 2013;56(10):512-25.

25. Martin SS, Blaha MJ, Elshazly MB, Toth PP, Kwiterovich PO, Blumenthal RS, Jones SR. Comparison of a novel method vs the Friedewald equation for estimating low-density lipoprotein cholesterol levels from the standard lipid profile. JAMA. 2013;310(19):2061-8.

26. Levey AS, Stevens LA, Schmid CH, Zhang YL, Castro AF, Feldman HI, Kusek JW, Eggers P, Van Lente F, Greene T. A new equation to estimate glomerular filtration rate. Ann Intern Med. 2009:150(9):604-12.

27. Drawz P, Rahman M. CHronic kidney disease. Ann Intern Med. 2015;162(11):1

28. Disease Kidney. Improving Global Outcomes (KDIGO) CKD-MBD Work Group: kDIGO clinical practice guideline for the diagnosis, evaluation, prevention, and treatment of Chronic Kidney Disease-Mineral and Bone Disorder (CKD-MBD). Kidney Int Suppl. 2009;113:S1-130.

29. Mast R, Jansen AD, Walraven I, Rauh SP, van der Heijden AA, Heine RJ Elders PJ, Dekker JM, Nijpels G, Hugtenburg JG. Time to insulin initiation and long-term effects of initiating insulin in people with type 2 diabetes mellitus: the Hoorn Diabetes Care System Cohort Study. Eur J Endocrinol. 2016:174(5):563-71.

30. Hope SV, Knight BA, Shields BM, Hill AV, Choudhary P, Strain WD, McDonald TJ, Jones AG. Random non-fasting C-peptide testing can identify patients with insulin-treated type 2 diabetes at high risk of hypoglycaemia. Diabetologia. 2018;61(1):66-74.

31. Zoppini G, Verlato G, Targher G, Casati S, Gusson E, Biasi V, Perrone F, Bonora $E$, Muggeo $M$. Is fasting glucose variability a risk factor for retinopathy in people with type 2 diabetes? Nutrition Metab Cardiovasc Dis. 2009;19(5):334-9.

32. Gorst C, Kwok CS, Aslam S, Buchan I, Kontopantelis E, Myint PK. Longterm glycemic variability and risk of adverse outcomes: a systematic review and meta-analysis. Diabetes Care. 2015;38:2354-69.

33. Lin CC, Yang CP, Li Cl, Liu CS, Chen CC, Lin WY, Hwang KL, Yang SY, Li TC. Visit-to-visit variability of fasting plasma glucose as predictor of ischemic stroke: competing risk analysis in a national cohort of Taiwan Diabetes Study. BMC Med. 2014;12:165

34. Penno G, Solini A, Zoppini G, Orsi E, Fondelli C, Zerbini G, Morano S, Cavalot F, Lamacchia O, Trevisan R, et al. Hemoglobin A1c variability as an independent correlate of cardiovascular disease in patients with type 2 diabetes: a cross-sectional analysis of the renal insufficiency and cardiovascular events (RIACE) Italian multicenter study. Cardiovasc Diabetol. 2013;12:98

35. Sugawara A, Kawai K, Motohashi S, Saito K, Kodama S, Yachi Y, Hirasawa R, Shimano H, Yamazaki K, Sone H. HbA(1c) variability and the development of microalbuminuria in type 2 diabetes: tsukuba Kawai Diabetes Registry 2. Diabetologia. 2012;55(8):2128-31. 
36. Hsu CC, Chang HY, Huang MC, Hwang SJ, Yang YC, Lee YS, Shin SJ, Tai TY. HbA1c variability is associated with microalbuminuria development in type 2 diabetes: a 7-year prospective cohort study. Diabetologia. 2012;55(12):3163-72.

37. Cheng D, Fei Y, Liu Y, Li J, Xue Q, Wang X. HbA1C variability and the risk of renal status progression in Diabetes Mellitus: a meta-analysis. PLOS ONE. 2014;9:e115509.

38. Takao T, Matsuyama Y, Yanagisawa H, Kikuchi M, Kawazu S. Visit-tovisit variability in systolic blood pressure predicts development and progression of diabetic nephropathy, but not retinopathy, in patients with type 2 diabetes. J Diabetes Compl. 2014;28(2):185-90.

\section{Publisher's Note}

Springer Nature remains neutral with regard to jurisdictional claims in published maps and institutional affiliations.
Ready to submit your research? Choose BMC and benefit from:

- fast, convenient online submission

- thorough peer review by experienced researchers in your field

- rapid publication on acceptance

- support for research data, including large and complex data types

- gold Open Access which fosters wider collaboration and increased citations

- maximum visibility for your research: over $100 \mathrm{M}$ website views per year

At BMC, research is always in progress.

Learn more biomedcentral.com/submissions 